\title{
An hybrid mean value of quadratic Gauss sums and a sum analogous to Kloosterman sums
}

\author{
Xiaowei $\operatorname{Pan}^{1}$ and Han Zhang ${ }^{2^{*}}$
}

\section{"Correspondence:}

micohanzhang@gmail.com

${ }^{2}$ Department of Mathematics,

Northwest University, Xi'an, Shaanxi,

P.R. China

Full list of author information is

available at the end of the article

\begin{abstract}
The main purpose of this paper is, using the analytic methods and the properties of character sums, to study the computational problem of one kind of hybrid mean value involving the quadratic Gauss sums and a new sum analogous to Kloosterman sums, and to give an interesting hybrid mean value formula for it.
\end{abstract}

MSC: 11L03; 11L40

Keywords: quadratic Gauss sums; a sum analogous to Kloosterman sums; hybrid mean value; identity

\section{Introduction}

Let $q \geq 3$ be an integer, and let $\chi$ be a Dirichlet character $\bmod q$. Then for any integer $n$, the famous quadratic Gauss sums $G(\chi, n ; q)$ is defined as follows:

$$
G(\chi, n ; q)=\sum_{a=1}^{q} \chi(a) \cdot e\left(\frac{n a^{2}}{q}\right)
$$

where $e(y)=e^{2 \pi i y}$.

This sum plays a very important role in the study of analytic number theory, many famous number theoretic problems are closely related to it. For example, the distribution of primes, the Goldbach problem, the properties of Dirichlet $L$-functions are some good examples. About the arithmetic properties of $G(\chi, n ; q)$, some authors had studied it and obtained many interesting results. For example, if $q=p$ is a prime and $(p, n)=1$, then one can get the estimate $|G(\chi, n ; p)| \leq 2 \sqrt{p}$. Some other results can be found in references [1-6].

On the other hand, the classical Kloosterman sums $K(m, n ; q)$ is defined as

$$
K(m, n ; q)=\sum_{a=1}^{q-1} e^{\prime}\left(\frac{m a+n \bar{a}}{q}\right),
$$

where $\sum_{a=1}^{q-1}$ denotes the summation over all $1 \leq a \leq q$ such that $(a, q)=1$, and $\bar{a}$ denotes the solution of the congruence equation $a x \equiv 1 \bmod q$.

\section{Springer}

@2014 Pan and Zhang; licensee Springer. This is an Open Access article distributed under the terms of the Creative Commons Attribution License (http://creativecommons.org/licenses/by/2.0), which permits unrestricted use, distribution, and reproduction in any medium, provided the original work is properly cited. 
Now we define another sum analogous to Kloosterman sums as follows:

$$
S(\chi, q)=\sum_{a=1}^{q-1} \chi(a+\bar{a})
$$

In fact, this sum is a special case of the general character of polynomials, some related results can be found in $[7,8]$ and $[9,10]$.

The main purpose of this paper is using the analytic method and the properties of the character sums to study the hybrid mean value properties of $G(\chi, n ; p)$ and $S(\chi, p)$, and to give an interesting mean value formula. That is, we shall prove the following two conclusions.

Theorem 1 Let $p$ be an odd prime, $\chi$ be any non-principal even character $($ i.e. $\chi(-1)=1)$ $\bmod p$. Then for any integer $n$ with $(n, p)=1$, we have the identity

$$
\left|\sum_{a=1}^{p-1} \chi(a) \cdot e\left(\frac{n a^{2}}{p}\right)\right|^{2}=2 p+\bar{\chi}(2) \cdot\left(\frac{n}{p}\right) \cdot \tau\left(\chi_{2}\right) \cdot \sum_{a=1}^{p-1} \chi(a+\bar{a}),
$$

where $\left(\frac{*}{p}\right)=\chi_{2}$ denotes the Legendre symbol, and $\tau\left(\chi_{2}\right)=\sum_{a=1}^{p-1} \chi_{2}(a) \cdot e\left(\frac{a}{p}\right)$ denotes the classical Gauss sums with $\tau^{2}\left(\chi_{2}\right)=\left(\frac{-1}{p}\right) \cdot p$.

Theorem 2 Let $p$ be an odd prime with $p \equiv 3 \bmod 4$. Then for any integer $n$ with $(n, p)=1$, we have the identity

$$
\sum_{\chi \bmod p}^{\prime}\left|\sum_{a=1}^{p-1} \chi(a) \cdot e\left(\frac{n a^{2}}{p}\right)\right|^{2} \cdot\left|\sum_{a=1}^{p-1} \chi(a+\bar{a})\right|^{2}=(p-1) \cdot\left(3 p^{2}-6 p-1\right)
$$

where $\sum_{\chi \bmod p}^{\prime}$ denotes the summation over all even character $\bmod p$, i.e. $\chi(-1)=1$.

Some notes: Theorem 1 tells us that there exists a close relationship between $G(\chi, n ; p)$ and $S(\chi, p)$. That is, $|G(\chi, n ; p)|^{2}$ can be represented by $S(\chi, p)$.

Since for any odd character $\chi$ mod $p$, we have $G(\chi, n ; p)=S(\chi, p)=0$, we only discussed the summation for all even characters $\chi \bmod p$ in Theorem 2 .

If $p \equiv 1 \bmod 4$, then we cannot give a computational formula for the hybrid mean value in Theorem 2. In this case, the difficulty is that we cannot obtain an exact value for the behind formula (13). We hope that the interested reader will stay with us as we turn to further study.

For general integer $q \geq 3$, whether there exists a computational formula for the hybrid mean value

$$
\sum_{\chi \bmod q}^{\prime}\left|\sum_{a=1}^{q} \chi(a) \cdot e\left(\frac{n a^{2}}{q}\right)\right|^{2} \cdot\left|\sum_{a=1}^{q-1} \chi(a+\bar{a})\right|^{2}
$$

is an interesting open problem, where $n$ is any integer with $(n, q)=1$. 


\section{Several lemmas}

In this section, we shall give two simple lemmas, which are necessary in the proofs of our theorems. Hereinafter, we shall use many properties of character sums and Gauss sums, all of these can be found in references $[1,2]$ and [11]. First we have the following.

Lemma 1 Let $p$ be an odd prime, $\chi$ be any non-principal even character mod $p$. Then we have the identity

$$
\left|\sum_{a=1}^{p-1} \chi(a+\bar{a})\right|^{2}=2 p+\sum_{a=1}^{p-1} \chi(a) \sum_{b=1}^{p-1}\left(\frac{b(b-1)\left(a^{2} b-1\right)}{p}\right),
$$

where $\left(\frac{*}{p}\right)$ denotes the Legendre symbol.

Proof Let $a+\bar{a}=u$, then we have

$$
\begin{aligned}
\sum_{a=1}^{p-1} \chi(a+\bar{a}) & =\sum_{u=1}^{p-1} \chi(u) \quad \sum_{\substack{a=1 \\
a+\bar{a}=u \bmod p}}^{p-1} 1=\sum_{u=1}^{p-1} \chi(u) \sum_{\substack{a=1 \\
a^{2}-a u+1 \equiv 0 \bmod p}}^{p-1} 1 \\
& =\sum_{u=1}^{p-1} \chi(u) \quad \sum_{\substack{a=0 \\
(2 a-u)^{2} \equiv u^{2}-4 \bmod p}}^{p-1} 1=\sum_{u=1}^{p-1} \chi(u) \sum_{\substack{a=0 \\
a^{2} \equiv u^{2}-4 \bmod p}}^{p-1} 1 .
\end{aligned}
$$

Note that for any fixed integer $u^{2}-4$, the number of the solutions of the congruence equation $x^{2} \equiv u^{2}-4 \bmod p$ are $1+\left(\frac{u^{2}-4}{p}\right)$, so from (1) we have

$$
\begin{aligned}
\sum_{a=1}^{p-1} \chi(a+\bar{a}) & =\sum_{u=1}^{p-1} \chi(u)\left(1+\left(\frac{u^{2}-4}{p}\right)\right) \\
& =\sum_{u=1}^{p-1} \chi(u)\left(\frac{u^{2}-4}{p}\right)=\chi(2) \sum_{u=1}^{p-1} \chi(u)\left(\frac{u^{2}-1}{p}\right) .
\end{aligned}
$$

Now from (2) and the properties of reduced residue system $\bmod p$ we have

$$
\begin{aligned}
\left|\sum_{a=1}^{p-1} \chi(a+\bar{a})\right|^{2} & =\left|\sum_{u=1}^{p-1} \chi(u)\left(\frac{u^{2}-1}{p}\right)\right|^{2}=\sum_{a=1}^{p-1} \sum_{b=1}^{p-1} \chi(a \bar{b})\left(\frac{a^{2}-1}{p}\right)\left(\frac{b^{2}-1}{p}\right) \\
& =\sum_{a=1}^{p-1} \chi(a) \sum_{b=1}^{p-1}\left(\frac{a^{2} b^{2}-1}{p}\right)\left(\frac{b^{2}-1}{p}\right) \\
& =\sum_{a=1}^{p-1} \chi(a) \sum_{b=1}^{p-1}\left(1+\left(\frac{b}{p}\right)\right)\left(\frac{a^{2} b-1}{p}\right)\left(\frac{b-1}{p}\right) \\
& =\sum_{a=1}^{p-1} \chi(a) \sum_{b=1}^{p-1}\left(\frac{\left(a^{2} b-1\right)(b-1)}{p}\right) \\
& +\sum_{a=1}^{p-1} \chi(a) \sum_{b=1}^{p-1}\left(\frac{\left(a^{2} b-1\right) b(b-1)}{p}\right) .
\end{aligned}
$$


Note that $\chi(-1)=1$, from the properties of the complete residue system mod $p$ we also have

$$
\begin{aligned}
\sum_{a=1}^{p-1} \chi(a) \sum_{b=1}^{p-1}\left(\frac{\left(a^{2} b-1\right)(b-1)}{p}\right) & =\sum_{a=1}^{p-1} \chi(a) \sum_{b=0}^{p-1}\left(\frac{\left(2 a^{2} b-a^{2}-1\right)^{2}-\left(a^{2}-1\right)^{2}}{p}\right) \\
& =\sum_{a=1}^{p-1} \chi(a) \sum_{b=0}^{p-1}\left(\frac{b^{2}-\left(a^{2}-1\right)^{2}}{p}\right)
\end{aligned}
$$

and

$$
\sum_{a=1}^{p}\left(\frac{a^{2}+n}{p}\right)= \begin{cases}-1, & \text { if }(n, p)=1 \\ p-1, & \text { if }(n, p)=p .\end{cases}
$$

(This formula can be found in Hua's book [11], Section 7.8, Theorem 8.2.)

Combining (4) and (5) we can deduce the identity

$$
\sum_{a=1}^{p-1} \chi(a) \sum_{b=1}^{p-1}\left(\frac{\left(a^{2} b-1\right)(b-1)}{p}\right)=2(p-1)-\sum_{a=2}^{p-2} \chi(a)=2 p .
$$

Now Lemma 1 follows from (3) and (6).

Lemma 2 Let $p$ be an odd prime, $\chi$ be any non-principal even character mod $p$. Then for any integer $m$ with $(m, p)=1$, we have the identity

$$
\left|\sum_{a=1}^{p-1} \chi(a) \cdot e\left(\frac{m a^{2}}{p}\right)\right|^{2}=2 p+\left(\frac{m}{p}\right) \cdot \tau\left(\chi_{2}\right) \cdot \sum_{a=1}^{p-1} \chi(a)\left(\frac{a^{2}-1}{p}\right)
$$

where $\chi_{2}=\left(\frac{*}{p}\right)$ denotes the Legendre symbol with $\tau^{2}\left(\chi_{2}\right)=\left(\frac{-1}{p}\right) \cdot p$.

Proof If $(m, p)=1$, then from the properties of Gauss sums and quadratic residue mod $p$ we have

$$
\begin{aligned}
\sum_{a=0}^{p-1} e\left(\frac{m a^{2}}{p}\right) & =1+\sum_{a=1}^{p-1} e\left(\frac{m a^{2}}{p}\right)=1+\sum_{a=1}^{p-1}\left(1+\left(\frac{a}{p}\right)\right) \cdot e\left(\frac{m a}{p}\right) \\
& =\sum_{a=0}^{p-1} e\left(\frac{m a}{p}\right)+\sum_{a=1}^{p-1}\left(\frac{a}{p}\right) \cdot e\left(\frac{m a}{p}\right) \\
& =\left(\frac{m}{p}\right) \sum_{a=1}^{p-1}\left(\frac{a}{p}\right) \cdot e\left(\frac{a}{p}\right)=\left(\frac{m}{p}\right) \cdot \tau\left(\chi_{2}\right) .
\end{aligned}
$$

Since $\chi$ is a non-principal even character $\bmod p$, so from identity (7) and the definition of $G(\chi, m ; p)$ we have

$$
\begin{aligned}
|G(\chi, m ; p)|^{2} & =\sum_{a=1}^{p-1} \sum_{b=1}^{p-1} \chi(a \bar{b}) \cdot e\left(\frac{m a^{2}-m b^{2}}{p}\right) \\
& =\sum_{a=1}^{p-1} \chi(a) \cdot \sum_{b=1}^{p-1} e\left(\frac{m b^{2}\left(a^{2}-1\right)}{p}\right)=\sum_{a=1}^{p-1} \chi(a) \cdot\left(\sum_{b=0}^{p-1} e\left(\frac{m b^{2}\left(a^{2}-1\right)}{p}\right)-1\right)
\end{aligned}
$$




$$
\begin{aligned}
& =2(p-1)+\sum_{a=2}^{p-2} \chi(a) \cdot\left(\sum_{b=0}^{p-1} e\left(\frac{m b^{2}\left(a^{2}-1\right)}{p}\right)-1\right) \\
& =2(p-1)-\sum_{a=2}^{p-2} \chi(a)+\tau\left(\chi_{2}\right) \cdot \sum_{a=2}^{p-2} \chi(a)\left(\frac{m\left(a^{2}-1\right)}{p}\right) \\
& =2 p-\sum_{a=1}^{p-1} \chi(a)+\tau\left(\chi_{2}\right) \cdot \sum_{a=1}^{p-1} \chi(a)\left(\frac{m\left(a^{2}-1\right)}{p}\right) \\
& =2 p+\left(\frac{m}{p}\right) \cdot \tau\left(\chi_{2}\right) \cdot \sum_{a=1}^{p-1} \chi(a)\left(\frac{a^{2}-1}{p}\right) .
\end{aligned}
$$

This completes the proof of Lemma 2.

\section{Proof of the theorems}

In this section, we shall complete the proof of our theorems. First we prove Theorem 1 . In fact from (2) and Lemma 2 we may immediately deduce the identity

$$
\begin{aligned}
\left|\sum_{a=1}^{p-1} \chi(a) \cdot e\left(\frac{m a^{2}}{p}\right)\right|^{2} & =2 p+\left(\frac{m}{p}\right) \cdot \tau\left(\chi_{2}\right) \cdot \sum_{a=1}^{p-1} \chi(a)\left(\frac{a^{2}-1}{p}\right) \\
& =2 p+\bar{\chi}(2) \cdot\left(\frac{m}{p}\right) \cdot \tau\left(\chi_{2}\right) \cdot \sum_{a=1}^{p-1} \chi(a+\bar{a}) .
\end{aligned}
$$

This proves Theorem 1.

Now we prove Theorem 2; from Lemma 1 and Lemma 2 we have

$$
\begin{aligned}
& \sum_{\chi \bmod p}^{\prime}\left|\sum_{a=1}^{p-1} \chi(a) \cdot e\left(\frac{n a^{2}}{p}\right)\right|^{2} \cdot\left|\sum_{a=1}^{p-1} \chi(a+\bar{a})\right|^{2} \\
& =\left|\sum_{a=1}^{p-1} \chi_{0}(a) \cdot e\left(\frac{n a^{2}}{p}\right)\right|^{2} \cdot\left|\sum_{a=1}^{p-1} \chi_{0}(a+\bar{a})\right|^{2} \\
& +\sum_{\chi \bmod }^{\prime}\left(2 p+\left(\frac{n}{p}\right) \cdot \tau\left(\chi_{2}\right) \cdot \sum_{a=1}^{p-1} \chi(a)\left(\frac{a^{2}-1}{p}\right)\right) \\
& \quad \times\left(2 p+\sum_{a=1}^{p-1} \chi(a) \sum_{b=1}^{p-1}\left(\frac{b(b-1)\left(a^{2} b-1\right)}{p}\right)\right) .
\end{aligned}
$$

If $p \equiv 3 \bmod 4$, then we have $\left(\frac{-1}{p}\right)=-1$ and

$$
\begin{aligned}
& \left|\sum_{a=1}^{p-1} \chi_{0}(a) \cdot e\left(\frac{n a^{2}}{p}\right)\right|^{2}=\left|\sum_{a=0}^{p-1} e\left(\frac{n a^{2}}{p}\right)-1\right|^{2}=\left|\chi_{2}(n) \tau\left(\chi_{2}\right)-1\right|^{2}=p+1, \\
& \left|\sum_{a=1}^{p-1} \chi_{0}(a+\bar{a})\right|^{2}=\left(\sum_{a=1}^{p-1} 1\right)^{2}=(p-1)^{2} .
\end{aligned}
$$


Note that the identity

$$
\sum_{\chi \bmod p}^{\prime} \chi(a)= \begin{cases}\frac{p-1}{2}, & \text { if } a \equiv \pm 1 \bmod p \\ 0, & \text { otherwise }\end{cases}
$$

from (5) we have

$$
\begin{aligned}
& \sum_{\substack{\chi \bmod \\
\chi \neq \chi_{0}}} \sum_{a=1}^{p-1} \chi(a)\left(\frac{a^{2}-1}{p}\right)=\sum_{\chi \bmod } \sum_{p}^{p-1} \chi(a)\left(\frac{a^{2}-1}{p}\right)-\sum_{a=1}^{p-1}\left(\frac{a^{2}-1}{p}\right) \\
& =-\sum_{a=0}^{p-1}\left(\frac{a^{2}-1}{p}\right)+\left(\frac{-1}{p}\right)=1+\left(\frac{-1}{p}\right)=0 ; \\
& \sum_{\chi \bmod p} \sum_{a=1}^{p-1} \chi(a) \sum_{b=1}^{p-1}\left(\frac{b(b-1)\left(a^{2} b-1\right)}{p}\right) \\
& \quad=\sum_{\chi \bmod p}^{\prime} \sum_{a=1}^{p-1} \chi(a) \sum_{b=1}^{p-1}\left(\frac{b(b-1)\left(a^{2} b-1\right)}{p}\right)-\sum_{a=1}^{p-1} \sum_{b=1}^{p-1}\left(\frac{b(b-1)\left(a^{2} b-1\right)}{p}\right) \\
& \quad=(p-1) \cdot \sum_{b=1}^{p-1}\left(\frac{b(b-1)(b-1)}{p}\right)-\sum_{b=1}^{p-1}\left(\frac{b-1}{p}\right) \sum_{a=1}^{p-1}\left(\frac{a^{2}-\bar{b}}{p}\right) \\
& \quad=(p-1) \cdot \sum_{b=2}^{p-1}\left(\frac{b}{p}\right)-\sum_{b=1}^{p-1}\left(\frac{b-1}{p}\right)\left(-1-\left(\frac{-\bar{b}}{p}\right)\right) \\
& \quad 1-p-2\left(\frac{-1}{p}\right)=-(p-3) .
\end{aligned}
$$

Note that $\left(\frac{-1}{p}\right)=-1$ and

$$
\begin{aligned}
& \sum_{a=1}^{p-1} \sum_{b=1}^{p-1}\left(\frac{\left(a^{2}-1\right)\left(b^{2}-1\right)\left(a^{2} b^{2}-1\right)}{p}\right) \\
& =\sum_{a=1}^{p-1} \sum_{b=1}^{p-1}\left(\frac{\left(a^{2} \bar{b}^{2}-1\right)\left(b^{2}-1\right)\left(a^{2}-1\right)}{p}\right) \\
& =\sum_{a=1}^{p-1} \sum_{b=1}^{p-1}\left(\frac{\left(a^{2}-b^{2}\right)\left(b^{2}-1\right)\left(a^{2}-1\right)}{p}\right) \\
& =-\sum_{a=1}^{p-1} \sum_{b=1}^{p-1}\left(\frac{\left(b^{2}-a^{2}\right)\left(b^{2}-1\right)\left(a^{2}-1\right)}{p}\right) \\
& =-\sum_{a=1}^{p-1} \sum_{b=1}^{p-1}\left(\frac{\left(b^{2} a^{2}-a^{2}\right)\left(b^{2} a^{2}-1\right)\left(a^{2}-1\right)}{p}\right) \\
& =-\sum_{a=1}^{p-1} \sum_{b=1}^{p-1}\left(\frac{\left(a^{2}-1\right)\left(b^{2}-1\right)\left(a^{2} b^{2}-1\right)}{p}\right),
\end{aligned}
$$


so that we have the identities

$$
\sum_{a=1}^{p-1} \sum_{b=1}^{p-1}\left(\frac{\left(a^{2}-1\right)\left(b^{2}-1\right)\left(a^{2} b^{2}-1\right)}{p}\right)=0
$$

and

$$
\begin{aligned}
\sum_{\substack{\chi \bmod p \\
\chi \neq \chi_{0}}}^{\prime}\left(\sum_{a=1}^{p-1} \chi(a) \sum_{b=1}^{p-1}\left(\frac{b(b-1)\left(a^{2} b-1\right)}{p}\right)\right)\left(\sum_{a=1}^{p-1} \chi(a)\left(\frac{a^{2}-1}{p}\right)\right) \\
=\sum_{\chi \bmod p}^{\prime}\left(\sum_{a=1}^{p-1} \chi(a) \sum_{b=1}^{p-1}\left(\frac{b(b-1)\left(a^{2} b-1\right)}{p}\right)\right)\left(\sum_{c=1}^{p-1} \chi(c)\left(\frac{c^{2}-1}{p}\right)\right) \\
\quad-\left(\sum_{a=1}^{p-1} \sum_{b=1}^{p-1}\left(\frac{b(b-1)\left(a^{2} b-1\right)}{p}\right)\right)\left(\sum_{a=1}^{p-1}\left(\frac{a^{2}-1}{p}\right)\right) \\
=(p-1) \cdot \sum_{a=1}^{p-1} \sum_{b=1}^{p-1}\left(\frac{b(b-1)\left(a^{2} b-1\right)}{p}\right)\left(\frac{\bar{a}^{2}-1}{p}\right)-2-2\left(\frac{-1}{p}\right) \\
=-(p-1) \cdot \sum_{a=1}^{p-1} \sum_{b=1}^{p-1}\left(1+\left(\frac{b}{p}\right)\right)\left(\frac{(b-1)\left(a^{2} b-1\right)}{p}\right)\left(\frac{a^{2}-1}{p}\right) \\
\quad+(p-1) \cdot \sum_{a=1}^{p-1} \sum_{b=1}^{p-1}\left(\frac{(b-1)\left(a^{2} b-1\right)}{p}\right)\left(\frac{a^{2}-1}{p}\right) \\
=-(p-1) \cdot \sum_{a=1}^{p-1} \sum_{b=1}^{p-1}\left(\frac{\left(a^{2}-1\right)\left(b^{2}-1\right)\left(a^{2} b^{2}-1\right)}{p}\right) \\
\quad+(p-1) \cdot \sum_{a=1}^{p-1}\left(\frac{a^{2}-1}{p}\right) \sum_{b=0}^{p-1}\left(\frac{\left(2 a^{2} b-a^{2}-1\right)^{2}-\left(a^{2}-1\right)^{2}}{p}\right) \\
=(p-1) \cdot \sum_{a=1}^{p-1}\left(\frac{a^{2}-1}{p}\right) \sum_{b=0}^{p-1}\left(\frac{b^{2}-\left(a^{2}-1\right)^{2}}{p}\right)=0 . \\
=
\end{aligned}
$$

Combining (8)-(12) and (14) we may immediately deduce

$$
\begin{aligned}
& \sum_{\chi \bmod p}^{\prime}\left|\sum_{a=1}^{p-1} \chi(a) \cdot e\left(\frac{n a^{2}}{p}\right)\right|^{2} \cdot\left|\sum_{a=1}^{p-1} \chi(a+\bar{a})\right|^{2} \\
& =\left|\sum_{a=1}^{p-1} \chi_{0}(a) \cdot e\left(\frac{n a^{2}}{p}\right)\right|^{2} \cdot\left|\sum_{a=1}^{p-1} \chi_{0}(a+\bar{a})\right|^{2} \\
& +\sum_{\chi \bmod p}^{\prime}\left(2 p+\left(\frac{n}{p}\right) \cdot \tau\left(\chi_{2}\right) \cdot \sum_{a=1}^{p-1} \chi(a)\left(\frac{a^{2}-1}{p}\right)\right) \\
& \quad \times\left(2 p+\sum_{a=1}^{p-1} \chi(a) \sum_{b=1}^{p-1}\left(\frac{b(b-1)\left(a^{2} b-1\right)}{p}\right)\right)
\end{aligned}
$$




$$
\begin{aligned}
& =(p+1)(p-1)^{2}+4 p^{2}\left(\frac{p-1}{2}-1\right)-2 p(p-3) \\
& =3 p^{3}-9 p^{2}+5 p+1=(p-1) \cdot\left(3 p^{2}-6 p-1\right) .
\end{aligned}
$$

This completes the proof of our theorems.

\section{Competing interests}

The authors declare that they have no competing interests.

\section{Authors' contributions}

All authors contributed equally to the writing of this paper. All authors read and approved the final manuscript.

\section{Author details}

${ }^{1}$ Department of Health Management, Xi'an Medical University, Xi'an, P.R. China. ${ }^{2}$ Department of Mathematics, Northwest University, Xi'an, Shaanxi, P.R. China.

\section{Acknowledgements}

The authors would like to thank the referees for their very helpful and detailed comments, which have significantly improved the presentation of this paper. This work is supported by the P.E.D. (2013JK0561) and N.S.F. (11371291) of P.R. China.

Received: 12 February 2014 Accepted: 17 March 2014 Published: 28 Mar 2014

\section{References}

1. Apostol, TM: Introduction to Analytic Number Theory. Springer, New York (1976)

2. Chengdong, P, Chengbiao, P: Goldbach Conjecture. Science Press, Beijing (1992)

3. Ireland, K, Rosen, M: A Classical Introduction to Modern Number Theory, pp. 204-207. Springer, New York (1982)

4. Cochrane, T, Pinner, C: A further refinement of Mordell's bound on exponential sums. Acta Arith. 116, 35-41 (2005)

5. Evans, JW, Gragg, WB, LeVeque, RJ: On least squares exponential sum approximation with positive coefficients. Math. Comput. 34(149), 203-211 (1980)

6. Williams, KS: Exponential sums over GF(2n). Pac. J. Math. 40, 511-519 (1972)

7. Burgess, DA: On Dirichlet characters of polynomials. Proc. Lond. Math. Soc. 13, 537-548 (1963)

8. Granville, A, Soundararajan, K: Large character sums: pretentious characters and the Pólya-Vinogradov theorem. J. Am. Math. Soc. 20, 357-384 (2007)

9. Zhang, W, Yi, Y: On Dirichlet characters of polynomials. Bull. Lond. Math. Soc. 34, 469-473 (2002)

10. Zhang, W, Yao, W: A note on the Dirichlet characters of polynomials. Acta Arith. 115, 225-229 (2004)

11. Hua, LK: Introduction to Number Theory. Science Press, Beijing (1979)

\section{Submit your manuscript to a SpringerOpen ${ }^{\circ}$ journal and benefit from:}

- Convenient online submission

Rigorous peer review

- Immediate publication on acceptance

- Open access: articles freely available online

- High visibility within the field

- Retaining the copyright to your article 